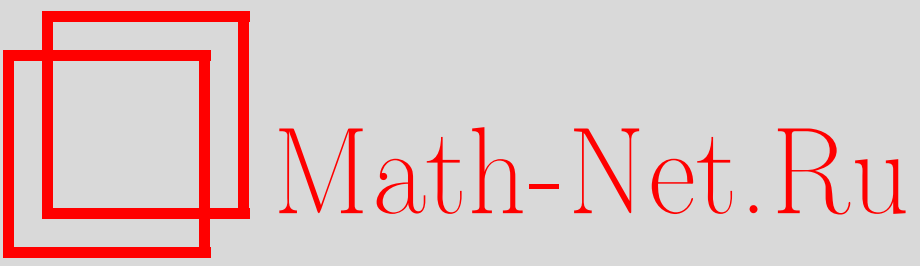

А. Е. Винн, Гиперболические модель Гейзенберга и сигма-модель в $(1+1)$ измерениях, ТМФ, 2000, том 122, номер 2, 310-320

DOI: https://doi.org/10.4213/tmf1934

Использование Общероссийского математического портала Math-Net.Ru подразумевает, что вы прочитали и согласны с пользовательским соглашением

http: //www. mathnet.ru/rus/agreement

Параметры загрузки:

IP : 3.95 .254 .165

26 апреля 2023 г., 12:32:12 
ТЕОРЕТИЧЕСКАЯ

И МАТЕМАТИЧЕСКАЯ

ФИЗИКА

Том 122, № 2

февраль, 2000

(C) 2000 г.

А. Е. Винн*

\section{ГИПЕРБОЛИЧЕСКИЕ МОДЕЛЬ ГЕЙЗЕНБЕРГА И СИГМА-МОДЕЛЬ В $(1+1)$ ИЗМЕРЕНИЯХ}

Гиперболические варианты интегрируемых $(1+1)$-мерных ферромагнитной модели Гейзенберга и сигма-модели обсуждаются в контексте топологических решений, классифицируемых с помощью целого числа "намоток". Представлены некоторые явные решения, исследовано существование определенных классов таких наматывающих (winding) решений.

\section{1. ВВЕДЕНИЕ}

Классическая модель Гейзенберга и нелинейная сигма-модель - хорошо известные $(1+1)$-мерные интегрируемые системы, которые исследовались для случаев как компактных, так и некомпактных пространств [1-12]. Эти модели важны как с математической, так и с физической точек зрения, поскольку, например, их классическое решение есть просто гармоническое отображение на (псевдо-)риманово многообразие [6]. Известно также, что модель Гейзенберга калибровочно-эквивалентна нелинейным уравнениям Шредингера типа притяжения (в компактном случае) [8] или отталкивания (для некомпактного полевого пространства) [3], в то время как редукция некомпактной $O(2,1)$-сигма-модели эквивалентна уравнению Лиувилля [10]. Модель Гейзенберга описывает (например, в компактном случае) классический спин $\vec{S}$, распределенный вдоль линии, т.е. непрерывный одномерный ферромагнетик. Сигма-модели на некомпактных многообразиях используются в релятивистской теории струн [10] и в теории гравитации $[12]$.

Ниже мы продолжаем начатое в работе [2] обсуждение решений гиперболической $(1+1)$-мерной модели Гейзенберга (ГМГ) и гиперболической сигма-модели (ГСМ), классифицированных с помошью целого числа намоток. Поле $\vec{\psi}(t, x)=\left(\psi^{1}, \psi^{2}, \psi^{3}\right)$ принимает значения на однополостном гиперболоиде $H^{2}$ в пространстве $\mathbb{R}^{2+1}$ и удовлетворяет ограничению

$$
\eta_{a b} \psi^{a} \psi^{b}=1
$$

где $\eta_{a b}=\operatorname{diag}(1,1,-1)$. Переменная $t \in \mathbb{R}$ означает время, а $x \in X$ - пространственная переменная. В случае $X=\mathbb{R}$ на поле накладывается граничное условие $\vec{\psi}(t, \infty)=$

* Department of Mathematical Sciences, University of Durham, Durham, UK. E-mail: A.E.Winn@durham.ac.uk 
$\vec{\psi}(t,-\infty)$, а в случае $X=S^{1}$ поле $\vec{\psi}$ периодично по $x$. Если метрика на гиперболоиде (1) индуцируется тензором $\eta_{a b}$, то многообразие является симметричным пространством $S O(2,1) / S O(1,1)$. Его фундаментальная группа совпадает с группой целых чисел $\mathbb{Z}$. Поэтому для каждого фиксированного $t$ функция $\vec{\psi}$ есть непрерывное отображение кольца на гиперболоид $H^{2}$ и, следовательно, имеет число намоток $N \in \mathbb{Z}$, не зависящее от $t$. Поле $\vec{\psi}$ можно представить в виде замкнутой струны, намотанной вокруг гиперболоида и эволюционируюшей во времени.

Модель Гейзенберга получается при рассмотрении плотности гамильтониана

$$
\mathcal{H}=\frac{1}{2} \eta_{a b} \psi_{x}^{a} \psi_{x}^{b}
$$

(индекс $x$ означает дифференцирование по $x$ ) со скобками Пуассона

$$
\left\{\psi^{a}(x), \psi^{b}(y)\right\}=-\delta(x-y) \epsilon^{a b c} \eta_{c d} \psi^{d}(x) .
$$

Гамильтониан

$$
H=\frac{1}{2} \int_{X} \mathcal{H} d x
$$

не является положительно-определенным из-за индефинитности метрики в полевом пространстве. Уравнения движения для этой системы заданы соотношениями

$$
\frac{\partial}{\partial t} \psi^{a}(x)=-\epsilon_{b c}^{a} \frac{\partial^{2} \psi^{b}}{\partial x^{2}} \psi^{c} .
$$

Сигма-модель определяется плотностью лагранжиана

$$
\mathcal{L}=\frac{1}{2} \eta^{\mu \nu} \psi_{\mu}^{a} \psi_{\nu}^{b} \eta_{a b}
$$

и уравнениями движения

$$
\left(\psi_{\mu \nu}^{a}+\psi^{a}\left(\psi_{\mu}^{b} \psi_{\nu}^{c}\right) \eta_{b c}\right) \eta^{\mu \nu}=0
$$

причем здесь и далее $\eta^{\mu \nu}=\operatorname{diag}(1,-1)$ и $x^{\mu}=(t, x)$. Обе модели в $(1+1)$ измерениях интегрируемы в том смысле, что для каждой модели сушествует подходящая пара Лакса, которую можно получить аналитическим продолжением из аналогичной системы $S^{2}$.

\section{2. ВОЛНЫ ТРАНСЛЯЦИЙ В ГИПЕРБОЛИЧЕСКОЙ МОДЕЛИ ГЕЙЗЕНБЕРГА}

Гиперболоид может быть параметризован полярными углами $\theta$ и $\phi$ так, что

$$
\psi^{a}=(\operatorname{ch} \theta \cos \phi, \operatorname{ch} \theta \sin \phi, \operatorname{sh} \theta)
$$

Уравнение (5) в этом случае будет эквивалентно уравнениям

$$
\begin{aligned}
\theta_{t} & =2(\operatorname{sh} \theta) \theta_{x} \phi_{x}+(\operatorname{ch} \theta) \phi_{x x}, \\
\phi_{t} & =(\operatorname{sech} \theta) \theta_{x x}+(\operatorname{sh} \theta) \phi_{x}^{2} .
\end{aligned}
$$


В работе [2] было показано сушествование некоторых простых статических и зависящих от времени "наматывающих" (winding) решений для этой модели. Здесь мы продолжим поиск "наматывающих" решений типа волны трансляций. Введем функции $f(\xi)=$ $\theta(t, x)$ и $g(\xi)+c t=\phi(t, x)$, где характеристическая переменная $\xi=x-v t, v-$ скорость волны, а взаимодействия с внешними магнитными полями вводятся через константу $c$. Подстановка этих функций в (9) приводит к уравнению

$$
g^{\prime}=(k-v \operatorname{sh} f) \operatorname{sech}^{2} f,
$$

где $k$ постоянно, а штрих означает дифференцирование по $\xi$. Отсюда, в свою очередь, получается уравнение для $f$ :

$$
f^{\prime \prime}=\left(v^{2}-k^{2}\right) \text { th } f \operatorname{sech}^{2} f+k v \operatorname{sech} f\left(1-2 \operatorname{sech}^{2} f\right)+c \operatorname{ch} f
$$

Первый интеграл

$$
f^{\prime 2}=\frac{\left(v^{2}-k^{2}\right) \operatorname{sh}^{2} f-2 k v \operatorname{sh} f+2 c \operatorname{sh} f \operatorname{ch}^{2} f+2 Q \operatorname{ch}^{2} f}{\operatorname{ch}^{2} f}
$$

где $Q$ постоянно, может быть упрошен принятием обозначения $p=\operatorname{sh} f$, так что

$$
\frac{p^{\prime 2}}{2}=c p^{3}+\frac{p^{2}}{2}\left(v^{2}-k^{2}+2 Q\right)+p(c-k v)+Q=P(p),
$$

где $k, v, c, Q$ - четыре действительных параметра. Для получения наматывающего решения надо, чтобы функция $f(\xi)$ была в некотором смысле периодична по $x$ (в зависимости от граничных условий) и чтобы интеграл по области $X$ от (10) был конечным и кратным $2 \pi$, т.е.

$$
\Delta \phi=\int_{X} g^{\prime} d x=\int_{X} \frac{k-v p}{1+p^{2}} d x=2 \pi N,
$$

где $N \in \mathbb{Z}$. Рассматривая случай $c=0$, т.е. случай отсутствия внешнего поля, получаем

$$
\frac{p^{\prime 2}}{2}=\frac{\alpha}{2} p^{2}-k v p+Q=P(p),
$$

где $\alpha=v^{2}-k^{2}+2 Q$. Для наматывающего решения необходимы ограничения $\alpha<0$ и $Q>k^{2} v^{2} / 2 \alpha$, так что функция $P(p)$ положительна и ограничена между двумя действительными нулями. Переопределение констант и интегрирование приводят к решению

$$
p(\xi)=p_{0}+\frac{\sqrt{2 A}}{N} \sin \left[N\left(\xi-\xi_{0}\right)\right],
$$

где $A>0, N(\neq 0) \in \mathbb{Z}, p_{0}$ и $\xi_{0}$ - постоянные. Это решение $p(\xi)=\operatorname{sh} f$ периодично с периодом $2 \pi$ и представляет собой вариант волны трансляций статического решения (13) из работы [2]. То, что это наматывающее решение, можно увидеть следующим образом. Принимая $N=1$ (обобшение для $|N|>1$ достаточно просто) и полагая $\alpha=-1$ (тем самым исключая $Q$ ), при $\xi_{0}=0$ можно переписать решение (13) в через наши исходные константы $k, v$ :

$$
p(\xi)=-k v+\sqrt{\left(v^{2}+1\right)\left(k^{2}-1\right)} \sin \xi
$$


Это решение имеет период $2 \pi$ и зависит от двух параметров $v \in \mathbb{R}$ и $k \geqslant 1$, так что:

1) если $v=0$, то $\Delta \phi=2 \pi$ для любых $k \geqslant 1$ (ср.с пунктом 3 ниже);

2 ) если $k=1$, то $\Delta \phi=2 \pi$ для любых $v$ (причем для этого наматывающего решения $p \equiv-v)$;

$3)$ если $v \neq 0$ и $k>1$, уравнение (10) сводится к уравнению

$$
g^{\prime}=\frac{1}{2}\left(\frac{v+i k}{k v+i-\gamma \sin \xi}+\frac{v-i k}{k v-i-\gamma \sin \xi}\right) .
$$

Интегрирование этого уравнения дает

$$
g(\xi)=\operatorname{Re}\left\{-\frac{i}{2}\left[\ln \left(\frac{\operatorname{tg} \frac{\xi}{2}-\frac{i \gamma+i k-v}{1+i k v}}{\operatorname{tg} \frac{\xi}{2}-\frac{i \gamma-i k+v}{1+i k v}}\right)-\ln \left(\frac{\operatorname{tg} \frac{\xi}{2}+\frac{i \gamma+i k+v}{1-i k v}}{\operatorname{tg} \frac{\xi}{2}+\frac{i \gamma-i k-v}{1-i k v}}\right)\right]+\Lambda\right\},
$$

где $\Lambda$ - константа интегрирования. Соотношение (15) можно записать в виде

$$
g(\xi)=\operatorname{Re}\left[-i \ln \left(\frac{\operatorname{tg} \frac{\xi}{2}+\frac{a}{b}}{\operatorname{tg} \frac{\xi}{2}+\frac{c}{b}}\right)+\Lambda\right],
$$

где $a=-i \gamma-i k+v, b=1+i k v, c=-i \gamma+i k-v$. Увидеть, что решение в этой форме является решением наматываюшего типа, не так просто. Однако можно показать, что (16) может быть переписано в виде

$$
g(\xi)=\operatorname{Re}\left[-i \ln \left(\frac{\operatorname{tg} \frac{\xi}{2}+\Omega}{-\operatorname{tg} \frac{\xi}{2}-\Omega^{-1}}\right)\right],
$$

где

$$
\Omega=\frac{v-i k-i \gamma}{1+i k v}\left(=\frac{a}{b}\right) .
$$

Такое решение относится к типу наматывающих, в чем можно убедиться, анализируя поведение функции

$$
\Xi(x)=\frac{\operatorname{tg} \frac{\xi}{2}+\Omega}{-\operatorname{tg} \frac{\xi}{2}-\Omega^{-1}}
$$

при изменении $x$ от $-\pi$ до $\pi$. Действительно, функция $\Xi(x)$ есть непрерывная функция $x$, не обрашающаяся в нуль при действительных $x$, и $\Xi \rightarrow-1$ при $x \rightarrow \pm \pi$, так что $\Xi$ имеет форму петли, начинающейся и заканчивающейся в -1 . Рассматривая в комплексном $\Xi$-пространстве разрез от начала координат вдоль отрицательной действительной оси, мы можем показать, что $\Xi$ однократно оборачивается вокруг начала координат, т.е. $\Delta \phi= \pm 2 \pi$. Для этого функция $\Xi$ должна пересекать положительную действительную ось один и только один раз, так что уравнение $\Xi=\bar{\Xi}$ должно иметь единственное действительное решение $x$. Это в самом деле так, поскольку равенство $\Xi=\Xi$ предполагает, что

$$
\frac{\operatorname{tg} \frac{\xi}{2}+\Omega}{-\operatorname{tg} \frac{\xi}{2}-\Omega^{-1}}=\frac{\operatorname{tg} \frac{\xi}{2}+\bar{\Omega}}{-\operatorname{tg} \frac{\xi}{2}-\bar{\Omega}^{-1}} \Longrightarrow \operatorname{tg} \frac{\xi}{2}=\frac{\bar{\Omega} \Omega^{-1}-\Omega \bar{\Omega}^{-1}}{\Omega+\bar{\Omega}^{-1}-\Omega^{-1}-\bar{\Omega}},
$$


поэтому

$$
\xi=2 \operatorname{tg}^{-1}\left[\frac{-2\left(v-k^{2} v-\gamma k v\right)\left(1+k^{2} v^{2}\right)}{\left(v-k^{2} v-\gamma k v\right)^{2}+\left(k+\gamma+k v^{2}\right)^{2}+\left(1+k^{2} v^{2}\right)^{2}}\right],
$$

чем и задается единственное и действительное решение $x$ для всех $k, v \in \mathbb{R}$. Тогда $\phi=$ $\pm 2 \pi$, т.е. решение (17) действительно есть наматьвающее решение для ГМГ, причем $X=S^{1}$ и $c=0$. Таким образом, (16) тоже является наматываюшим решением.

Пытаясь найти более обшее решение, рассмотрим другой пример и положим, что $c=$ 1. В результате подстановок

$$
\alpha=\frac{1}{6}\left(k^{2}-v^{2}+2 Q\right), \quad \Delta=\alpha^{2}-\frac{1}{3}(1-k v)
$$

уравнение (11) преобразуется к виду

$$
P(p)=\frac{p^{\prime 2}}{2}=p^{3}-3 p^{2} \alpha+3\left(\alpha^{2}-\Delta\right) p+Q
$$

включаюшему три действительных параметра $k, v, Q$. Чтобы решение уравнения (18) было волной трансляций наматывающего типа, функция $P(p)$ должна иметь три действительных нуля. При этом существует положительная ограниченная область, в которой решение может осциллировать. Этим накладывается требование $\Delta>0$. Выясним условия такого поведения для пространств $X=S^{1}$ и $X=\mathbb{R}$. Чтобы упростить рассмотрение, прежде всего отметим, что если функция $P(p)$, заданная согласно (18), имеет три действительных и несовпадаюших нуля $p_{1}>p_{2}>p_{3}$, то сушествует следуюшее решение, выражаюшееся через эллиптические функции:

$$
p(\xi)=p_{2}-\left(p_{2}-p_{3}\right) \mathrm{cn}^{2}\left(\left(\xi-\xi_{3}\right) \sqrt{\frac{p_{1}-p_{3}}{2}} \mid m\right),
$$

где параметр $m=\left(p_{2}-p_{3}\right) /\left(p_{1}-p_{3}\right)$. Заметим, что данное решение есть также решение типа волны трансляций для уравнения Кортевега-де Фриза [13] и при заданных значениях $p_{1}, p_{2}, p_{3}$ форма кноидальной волны может быть установлена как с помощью таблиц эллиптических функций Якоби, так и прямым вычислением. Чтобы выяснить, является ли данное решение решением наматываюшего типа, следует проинтегрировать (10) при $p(\xi)$, заданном в виде (19), что является непростой (а может быть, и невыполнимой) задачей. Поэтому мы рассматриваем предельные случаи, когда $m=0$ или $m=1$. При $m=0$ имеем $p_{2}=p_{3}$ и у функции $P(p)$ два нуля совпадают при отсутствии положительной ограниченной области, о которой говорилось выше. Тогда решение есть $p=p_{3}=\operatorname{sh} f$, и в этом случае функция

$$
g^{\prime}=\frac{k-v p_{3}}{1+p_{3}^{2}}=N
$$

постоянна, так что, если $N \in \mathbb{Z}$, восстанавливается наматываюшее решение

$$
\phi=N x+t
$$


из работы [2]. Другой предельный случай реализуется, когда функция $P(p)$ имеет один “двойной” и один изолированный нуль и область между ними положительна и ограничена. Если $p_{1}-$ двойной, а $p_{3}$ - изолированный нули, то эта ситуация соответствует волне трансляций в пространстве $X=\mathbb{R}$, где решение имеет минимум при $p=p_{3}$ (поскольку $\left.P^{\prime}\left(p_{3}\right)>0\right)$ и стремится к $p=p_{1}$ при $\xi \rightarrow \pm \infty$. Возврашаясь теперь к решению (19) и рассматривая предел, когда параметр

$$
m=\frac{p_{2}-p_{3}}{p_{1}-p_{3}}=1,
$$

мы видим, что точка $p_{2}$ должна совпадать с точкой $p_{1}$, что в точности соответствует описанной ситуации. Поскольку $\operatorname{cn}(u \mid m)=\operatorname{sech} u$ при $m=1$, решение (19) редуцируется к виду

$$
p(\xi)=p_{1}-\left(p_{1}-p_{3}\right) \operatorname{sech}^{2}\left(\left(\xi-\xi_{0}\right) \sqrt{\frac{p_{1}-p_{3}}{2}}\right) .
$$

Можно найти явные выражения для $p_{1}, p_{3}$, удовлетворяющие уравнению (18). Оказывается, однако, что это решение не является наматываюшим решением модели Гейзенберга. После подстановки (20) в (10) (например, для случая одного витка) получим

где

$$
\begin{aligned}
g^{\prime}= & \frac{k-v \gamma}{\gamma^{2}-1}+\Omega\left(\frac{1}{\lambda-\operatorname{ch} X}+\frac{1}{\lambda+\operatorname{ch} X}\right)+ \\
& +\bar{\Omega}\left(\frac{1}{\bar{\lambda}-\operatorname{ch} X}+\frac{1}{\bar{\lambda}+\operatorname{ch} X}\right)
\end{aligned}
$$

$$
X=\left(\xi-\xi_{0}\right), \quad \gamma=\frac{1}{6}\left(k^{2}-v^{2}+2 Q+4\right), \quad \Omega=\frac{(v-i k) \sqrt{\gamma+i}}{2 \sqrt{2}(\gamma+i)^{2}}, \quad \lambda=\sqrt{\frac{2}{\gamma+i}} .
$$

При интегрировании по области $X=\mathbb{R}$ соотношения (21) возникает расходимость, так что функция $\phi$ “оборачивается” бесконечное число раз вокруг гиперболоида $H^{2}$. Таким образом, это решение не является наматываюшим решением, поскольку число намоток $N$ должно быть конечным.

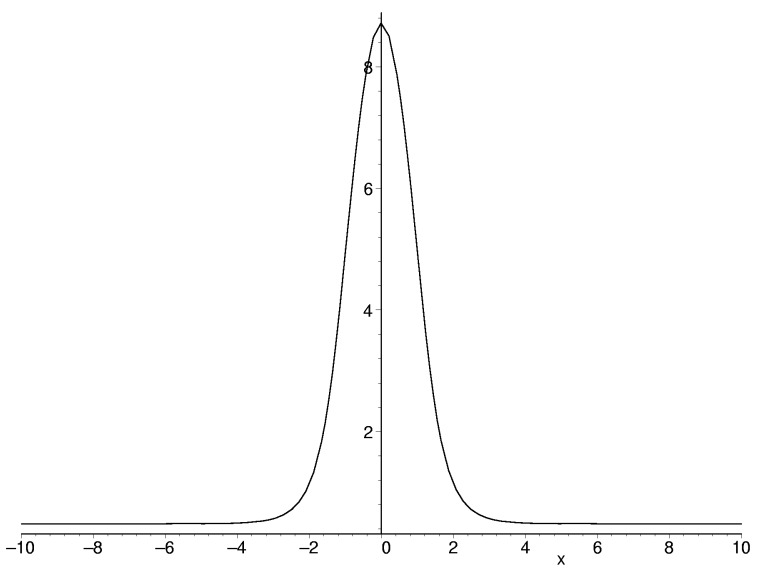


Заметим далее, что такое поведение проявляется и в соответствуюшей плотности гамильтониана. Например, рассмотрим простой случай $p_{1}=0$, так что $Q=0$, и для решения с одним витком $\sqrt{\Delta}=-2 / 3, v^{2}=2+\sqrt{5}$ и $p_{3}=-2$. Тогда плотность гамильтониана задается выражением

$$
\mathcal{H}=\frac{\left[1+2(2+\sqrt{5}) \operatorname{sech}^{2} X\right]^{2}-16(\sqrt{2+\sqrt{5}}) \operatorname{sech}^{4} X \operatorname{th}^{2} X}{(\sqrt{2+\sqrt{5}})\left(1+4 \operatorname{sech}^{4} X\right)}
$$

график которого показан на рисунке. Видно, что при $X=\xi-\xi_{0} \rightarrow \pm \infty$ плотность $\mathcal{H}$ не стремится к нулю, следовательно, гамильтониан бесконечен. Поскольку этот профиль плотности $\mathcal{H}$ обладает привлекательным однородным “горбом", возможно применение некоторой перенормировочной процедуры, которая устраняла бы эту бесконечность, однако здесь мы не будем исследовать данную возможность.

В работе [2] утверждалось, что для этой модели не существует решений наматывающего типа вида волны трансляций при $X=\mathbb{R}$. Сейчас мы покажем, что это действительно так. Для сушествования таких решений требуется, чтобы:

1) решение $p(\xi)$ было действительным и ограниченным, т.е. $P(p)=p^{\prime 2} \geqslant 0$ (из формы (11) $P(p)$ видно, что $p(\xi)$ меняется монотонно, пока $p^{\prime}(\xi)$ не обрашается в нуль);

2) $P(p)$ имело два действительных нуля: один двойной и один простой (соответственно $p_{0}$ и $p_{1}$, где решение $p(\xi)$ имеет минимум при $p=p_{1}$ и $p(x) \rightarrow p_{0}$ при $\left.x \rightarrow \pm \infty\right)$.

Вспомнив, что

$$
\Delta \phi=\int_{X} \frac{d g}{d \xi} d x=\int_{-\infty}^{\infty} \frac{k-v p(\xi)}{1+p^{2}(\xi)} d x
$$

необходимо потребовать, чтобы:

3) $p(\xi) \rightarrow k / v$ при $x \rightarrow \pm \infty$, где $k / v$ есть нуль для $d g / d \xi$, так что $p_{0}=k / v$ (см. пункт 2 выше);

4) $p^{\prime}(\xi) \rightarrow M$ ( $M-$ постоянная) при $x \rightarrow \pm \infty$. На самом деле, при $X=\mathbb{R}$ из утверждений 3 и 1 следует, что $M=0$, так что $P(p=k / v)=0$.

Заметив, что для $p_{0}=k / v$ функция $P(p)$ должна иметь форму

$$
P(p)=\left(p-\frac{k}{v}\right)^{2}(\alpha p-\beta)
$$

при некоторых $\alpha, \beta \in \mathbb{R}$, нам достаточно применить условие 4 к уравнению (11), чтобы показать, что условия 1-4 не могут быть удовлетворены для решения $p=\operatorname{sh} f$ ГМГ. Подстановка условия $P(p=k / v)=0$ в уравнение (11) дает

$$
\left(k^{2}+v^{2}\right)\left(2 Q v+2 c k-k^{2} v\right)=0 .
$$

Уравнение (23) имеет следующие решения:

$$
\begin{aligned}
v & = \pm i k, \\
v & =c=0, \\
v & =k=0, \\
k & =Q=0, \\
Q & =\frac{k}{2 v}(k v-2 c), \\
c & =\frac{v}{2 k}\left(k^{2}-2 Q\right) .
\end{aligned}
$$


Три первых случая могут не рассматриваться, поскольку требуются действительные ненулевые значения $v$. Случай (23г) приводит к функции

$$
P(p)=\frac{v^{2}}{2} p^{2}
$$

что вступает в противоречие с утверждением 2 (см. выше). Подстановка решения (23д) в (11) и сравнение получившегося уравнения с (22) допускают только $v= \pm i k$. Остается рассмотреть (23e). В этом случае находим, что или $v= \pm i k$, или $k^{2}=2 Q$. Из последнего выражения следует, что

$$
P(p)=\frac{1}{2}\left(p-\frac{k}{v}\right)^{2},
$$

что вновь противоречит утверждению 2 (см. выше). Таким образом, для значений параметров, удовлетворяющих (22), исключены все перечисленные выше возможности, поэтому не сушествует решения $p=\operatorname{sh} f$, удовлетворяюшего всем условиям $1-4$. Итак, показано, что, хотя для пространства $X=S^{1}$ решения наматывающего типа вида волн транслящий имеются, не сушествует таких решений для модели ГМГ, когда $X$ - действительное пространство $\mathbb{R}$.

\section{3. ГИПЕРБОЛИЧЕСКАЯ СИГМА-МОДЕЛЬ}

При параметризации (8) гиперболоида уравнения движения для ГСМ имеют вид

$$
\begin{gathered}
\theta_{t t}-\theta_{x x}=-\operatorname{ch} \theta \operatorname{sh} \theta\left(\phi_{t}^{2}-\phi_{x}^{2}\right), \\
\left(\phi_{t} \operatorname{ch}^{2} \theta\right)_{t}=\left(\phi_{x} \operatorname{ch}^{2} \theta\right)_{x}
\end{gathered}
$$

Энергия задается интегралом

$$
E=\int_{X} \epsilon d x
$$

где плотность энергии

$$
\epsilon=\operatorname{ch}^{2} \theta\left(\phi_{t}^{2}+\phi_{x}^{2}\right)-\left(\theta_{t}^{2}+\theta_{x}^{2}\right)
$$

Заметим, что это выражение содержит неопределенность, связанную с сигнатурой метрики на $H^{2}$.

При попытке найти решение с $\phi=N x$ оказывается, что $\theta$ зависит только от $t$, а в таком случае уравнения (24), (25) допускают решение

$$
\operatorname{th} \theta=\operatorname{sn}\left[\rho\left(t-t_{0}\right) \mid m\right]
$$

с $m=1-N^{2} / \rho^{2} \quad\left(\rho, t_{0}-\right.$ константы $), \rho>|N|$. Такое решение почти идентично решению уравнений для положительно-определенного случая [2]. Поскольку $\mathrm{th}^{-1} u \rightarrow \infty$ при $u \rightarrow 1$, можно видеть, что $\theta(t) \rightarrow \infty$ при $\operatorname{sn}\left(\rho\left(t-t_{0}\right) \mid m\right) \rightarrow 1$, т.е. функция $\theta(t)$ достигает бесконечных значений за конечное время. Для этого решения энергия имеет вид

$$
E=\int_{X}[A(\theta)-B(\theta)] d x
$$


где $A(\theta)=N^{2} \operatorname{ch}^{2} \theta$ и $B(\theta)=N^{2} \operatorname{sh}^{2} \theta+\rho^{2}$. Каждая из этих положительных функций обращается в бесконечность за конечное время (такая неустойчивость явно присутствует в решениях). Однако интересно заметить, что полная энергия является сохраняющейся (отрицательной) величиной. Такой же расчет для случая положительной определенности [2] показывает, что полная энергия положительна и ее значение действительно превышает значение нижней границы для энергии.

В поисках решений этой системы мы, как и в предыдушем разделе, обратимся к решениям вида волн трансляций для ГСМ. Уравнения движения в этом случае при $\theta=f(\xi)$, $\phi=g(\xi)+c t$ имеют вид

$$
\begin{aligned}
& f^{\prime \prime}\left(v^{2}-1\right)=-\left(g^{2}\left(v^{2}-1\right)-2 v c g^{\prime}+c^{2}\right) \operatorname{ch} f \operatorname{sh} f, \\
& g^{\prime \prime}\left(v^{2}-1\right)=-2\left(f^{\prime} g^{\prime}\left(v^{2}-1\right)-c v f^{\prime}\right) \text { th } f .
\end{aligned}
$$

Сначала опять рассмотрим случай $c=0$, когда

$$
\begin{aligned}
& f^{\prime \prime}=-g^{\prime 2} \operatorname{ch} f \operatorname{sh} f \\
& g^{\prime \prime}=-2 f^{\prime} g^{\prime} \operatorname{th} f
\end{aligned}
$$

Заметив, что

$$
\left(g^{\prime} \operatorname{ch}^{2} f\right)^{\prime}=2 f^{\prime} g^{\prime} \operatorname{ch} f \operatorname{sh} f+g^{\prime \prime} \operatorname{ch}^{2} f=0
$$

в силу (31), получаем следующие первые интегралы для $g$ и $f$ :

$$
\begin{aligned}
& g^{\prime}=B \operatorname{sech}^{2} f \\
& f^{\prime}=\sqrt{B^{2}-N^{2}-B^{2} \operatorname{th}^{2} f},
\end{aligned}
$$

где $B$ и $N$ - константы. Аналогичное решение для (13) может быть выведено из этих уравнений. Именно

$$
p(\xi)=\sqrt{\frac{B^{2}}{N^{2}}-1} \sin \left[N\left(\xi-\xi_{0}\right)\right],
$$

где снова $p=\operatorname{sh} f$, а $\xi_{0}$ - константа. Это решение - наматываюшее решение для ГСМ, что может быть показано так, как это было сделано для решения (13) в рамках ГМГ. На самом деле, поскольку ГСМ релятивистски инвариантна, приведенное выше решение также может быть получено из статического состояния, обсуждавшегося в работе [2].

Если $c \neq 0$, возникают следующие уравнения:

$$
\begin{aligned}
P(p)=p^{\prime 2}= & \frac{c^{2}}{\left(v^{2}-1\right)^{2}}\left[p^{4}+p^{2} \frac{6 c^{2}+4 Q\left(v^{2}-1\right)^{2}}{4 c^{2}}+\right. \\
& \left.+\frac{2 c^{2}+4 Q\left(v^{2}-1\right)^{2}+\left(4 R\left(v^{2}-1\right)^{2}-c v\right)^{2}}{4 c^{2}}\right], \\
g^{\prime}= & \frac{c v+2 R\left(v^{2}-1\right)+2 c v p^{2}}{1+p^{2}}
\end{aligned}
$$

где $R$ и $Q$ - константы. Для периодичности функции sh $f$ необходимо, чтобы функция $P(p)$ имела вид

$$
P(p)=\left(p^{2}-J^{2}\right)\left(p^{2}-K^{2}\right)
$$


при $J, K \in \mathbb{R}$, так что $P(p)$ положительна и ограничена в области между двумя нулями $p= \pm J$. Сравнение коэффициентов приводит к двум следующим уравнениям для $J$ и $K$ :

$$
\begin{gathered}
-\left(J^{2}+K^{2}\right)=\frac{6 c^{2}+4 Q\left(v^{2}-1\right)^{2}}{4 c^{2}} \\
J^{2} K^{2}=\frac{2 c^{2}+4 Q\left(v^{2}-1\right)^{2}+\left(4 R\left(v^{2}-1\right)^{2}-c v\right)^{2}}{4 c^{2}} .
\end{gathered}
$$

Если принять, что $q=Q\left(v^{2}-1\right)^{2}, r=R\left(v^{2}-1\right)^{2}, 4 \rho=4 r-c v$ и (без ограничения обшности) $c=1$, то отсюда получаются условия $q<-3 / 2,2 q>-\left(8 \rho^{2}+1\right)$ и $1-2 q \geqslant$ $8|\rho|$. Такие $q, \rho$ можно найти, так что решение для $p(\xi)$ после сдвига по $x$ можно записать через эллиптическую функцию

$$
p(\xi)=J_{\mathrm{sn}}\left[\frac{c K\left(\xi-\xi_{0}\right)}{v^{2}-1} \mid m\right]
$$

при $m=J^{2} / K^{2}$. Исследуя предельные случаи этого решения, находим, что $p(\xi) \equiv 0$ при $m=0$. В пределе $m=1$, если положить $K=J=p_{0}$, получим

$$
p(\xi)=p_{0} \text { th }\left[\frac{c p_{0}\left(\xi-\xi_{0}\right)}{v^{2}-1}\right] .
$$

В этом случае полином $P(p)$ положителен и ограничен между двумя двойными нулями $p= \pm p_{0}$, так что решение начинается с $-p_{0}$ при $x=-\infty$ и меняется до $p_{0}$ при $x=+\infty$, проходя только половину периода и эффективно выходя из пространства $x$. Поскольку это единственно возможный вариант для случая $X=\mathbb{R}$, то тем самым показано, что для этой модели с $X=\mathbb{R}$ наматывающих решений вида волн транслящий нет.

Если $P(p)$ имеет вид (37), для наматываюших решений, как обычно, требуется, чтобы $\Delta \phi=2 \pi N$. Тогда в описанном выше предельном случае $m=1$ следует ожидать, что $\Delta \phi$ окажется вдвое меньше этой величины, т.к. пройдена только половина периода. Однако это не так, поскольку

$$
g^{\prime}=\frac{c v}{v^{2}-1}+\frac{2 R\left(v^{2}-1\right)-c v}{2\left(v^{2}-1\right)\left(1+p_{0}^{2} \operatorname{th}^{2} X\right)},
$$

где

$$
X= \pm \frac{c p_{0}\left(\xi-\xi_{0}\right)}{v^{2}-1}
$$

и интеграл от этого выражения по всему пространству $\mathbb{R}$ очевидным образом расходится. Хотя эти рассуждения не могут считаться доказательством, они указывают на то, что обшее решение (39) не может быть наматываюшим решением. 


\section{4. ЗАКЛЮЧИТЕЛЬНЫЕ ЗАМЕЧАНИЯ}

Интегрируемые системы, которые допускают топологические решения, зависяшие от времени, встречаются сравнительно редко. Вероятно, единственным хорошо известным примером является случай уравнения синус-Гордон. Описанные в этой статье модели относятся к именно таким интегрируемым моделям. Нами показано, что, хотя для гиперболической модели Гейзенберга с $X=\mathbb{R}$ решений вида волн трансляций, классифицируемых с помощью целого числа намоток, не существует и такие решения едва ли существуют для ГСМ с таким же $X$, они существуют, если $X=S^{1}$. В действительности мы показали, что сушествуют решения для $X=S^{1}$, имеющие в обеих моделях одинаковую форму (но не совпадаюшие). Таким образом, может представлять интерес попытка осуществления какой-то формы интерполяции между решениями и, возможно, меж ду самими моделями.

Благодарности. Эта работа выполнена при поддержке EPSRC (Исследовательская студенческая стипендия). Автор благодарен профессору Р. С. Уорду за его терпение и руководство.

\section{Список литературы}

[1] L. A. Takhtajan. Phys. Lett. A. 1977. V. 64. № 2. P. 235.

[2] R. S. Ward, A. E. Winn. J. Phys. A. 1998. V. 31. P. L261.

[3] A. Kundu. Lett. Math. Phys. 1982. V. 6. P. 479.

[4] A. Kundu. J. Phys. A. 1986. V. 19. P. 1303.

[5] D. Lambert, B. Piette. Class. Q. Grav. 1988. V. 5. P. 307.

[6] J. P. Antoine, B. Piette. J. Math. Phys. 1988. V. 29. № 7. P. 1687.

[7] J. H. Lee, O. K. Pashaev. Abelian Gauge Theory and Integrable $\sigma$-Models. MIAS 97-3. Massachusets: MIAS, 1997.

[8] Л. А. Тахтаджян, Л. Д. Фаддеев. Гамильтонов подход в теории солитонов. М.: Наука, 1986.

[9] H. Eichenherr, J. Honerkamp. J. Math. Phys. 1981. V. 22. № 2. P. 374.

[10] H. J. de Vega, N. Sanchez. Phys. Rev. D. 1993. V. 47. № 8. P. 3394.

[11] J. Tjon, J. Wright. Phys. Rev. B. 1977. V. 15. № 7. P. 3470.

[12] P. O. Mazur. J. Phys. A. 1982. V. 15. P. 3173; Acta Phys. Polon. B. 1983. V. 14. P. 219; Phys. Lett. A. 1984. V. 100. P. 314.

[13] P. F. Drazin, R. S. Johnson. Solitons: an Introduction. Cambridge: Cambridge University Press, 1989. 